Article original

\title{
La prise en charge psychologique de fillettes victimes de violences sexuelles au Sud Kivu
}

\section{Evaluation of a therapeutic device for girls victims of sexual violence in South Kivu}

\author{
V. De Keyser ${ }^{\mathrm{a}, \mathrm{b}}$, C. de Kerchove ${ }^{\mathrm{b}}, \mathrm{C}$. Amisi $^{\mathrm{c}}$, S. Ntamwenge ${ }^{\mathrm{b}, \mathrm{c}}$, A. Blavier $^{\mathrm{a}, \mathrm{b}, *}$ \\ a Faculté de psychologie, logopédie et sciences de l'éducation, centre d'expertise en psychotraumatisme et psychologie légale, université de Liège, place des \\ Orateurs, 1 - bâtiment B33 - quartier Agora, 4000 Liège, Belgique \\ ${ }^{\mathrm{b}}$ ASBL Les Enfants de Panzi et d'Ailleurs, 4000 Liège, Belgique \\ ${ }^{\mathrm{c}}$ Hôpital de Panzi, Bukavu, République démocratique du Congo
}

\section{N F O A A R T I C L E}

\section{Mots clés :}

Traumatisme

Enfants

Centrafrique

Humanitaire

Dispositif thérapeutique

\section{R É S U M É}

Objectif. - Cet article évalue un dispositif de prise en charge psychologique de fillettes victimes de violences sexuelles dans trois villages différents autour de Bukavu (RDC).

Participants. - Cinquante-sept fillettes de moins de 10 ans ayant subi des violences sexuelles dans trois villages différents autour de Bukavu et suivies par l'association EPA et la Fondation Panzi.

Méthode. - Les trois échantillons d'enfants correspondant aux trois sites ont été testés afin de voir s'ils étaient comparables, une série de données concernant les fillettes a été recueillie afin de vérifier leur influence sur l'évolution de l'enfant et, finalement, un indice de fonctionnement global a été utilisé pour mesurer l'efficacité de la thérapie par le jeu.

Résultats. - Les trois échantillons d'enfants sont très semblables et donc comparables. Aucune variable n'est prédictive de l'évolution de l'enfant, une fois celles liée au site et à la présence du père exclues. La thérapie par le jeu n'a été totalement positive que pour un site, le plus sécurisé des trois.

Conclusion. - Il existe un facteur de contexte lié aux particularités du site ainsi qu'un facteur individuel ou intrafamilial qui influence l'évolution de l'enfant. La thérapie par le jeu n'a pas d'effet significatif sur ceux-ci même si elle permet de lutter contre la stigmatisation de ces fillettes.

(c) 2020 Elsevier Masson SAS. Tous droits réservés.

\section{A B S T R A C T}

Objective. - This article evaluates a psychological care program for girls who were victims of sexual violence in three different villages around Bukavu (DRC).

Participants. - Fifty-seven girls under 10 years of age who have suffered sexual violence in three different villages around Bukavu and followed by the EPA association ("Enfants de Panzi et d'Ailleurs") and the Panzi Foundation.

Method. - The three samples of children corresponding to the three villages were tested to ensure that they were comparable through different variables such as the father's presence, the age of the victims and the children' stigmatization. A series of data about the girls was collected and transformed into 13 variables in order to test their influence on the child's development. Finally, an overall functioning index was used to measure the effectiveness of play therapy. This global functioning index concerned five fields: feeling of security, health, cognitive development, family integration and community integration.

Results. - The three samples of children were very similar and therefore comparable. No variables were predictive of the child's development, once those related to the site and the presence of the father were excluded. However, a high-risk group of children in the three regions emerged from the analysis. Play

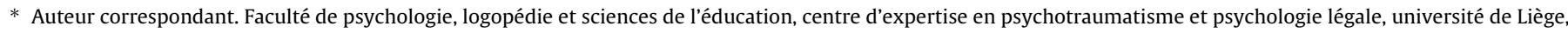
place des Orateurs, 1 - bâtiment B33 - quartier Agora, 4000 Liège, Belgique.

Adresse e-mail : Adelaide.Blavier@ulg.ac.be (A. Blavier).
} 
therapy was only totally positive for one site, the most secure of the three. Moreover, a group of highrisk children from all regions of origin was identified in the study and presented some very worrying characteristics.

Discussion. - The denial of young raped children allows criminals to go unpunished. Travelling in DCR is difficult for victims because of distance, cost and road insecurity; moreover, the number of victims is high, and it is thus difficult to create individual therapy. Moreover, as the community is an essential symbol, the use of group therapy, and in particular play therapy, is really appropriate instead of individual therapy. The mother-child relationship should be further investigated and could be an important variable for the high-risk group.

Conclusion. - There was a context factor related to the particularities of the site as well as an individual or intra-family factor that influences the child's development. Even if play therapy did not have a directly significant effect on children, it helped to combat the stigmatization of these girls. Moreover, play therapy may have an effect on variables other than those considered in this study or may be masked by mediating variables. Finally, a group of high-risk children that has been identified in the study presented worrying clinical data and particular features that are of great concern and will require special attention and care.

(c) 2020 Elsevier Masson SAS. All rights reserved.

\section{Contexte}

La région du Sud Kivu est excessivement riche du point de vue des ressources minières et fait donc l'objet de convoitises. Elle est devenue, suite aux blessures mal pansées de la guerre, à l'impunité des crimes enregistrés dans le rapport Mapping de l'Onu de 2010 [1] et à ses richesses à la portée de tous les prédateurs, une zone de nondroit où la violence s'est généralisée. Et dans son immense majorité, la population, loin de profiter de ces richesses, est dans un état de survie économique. La plupart des familles vivent avec moins de dix dollars par mois pour des fratries d'une dizaine d'enfants. La violence qu'on enregistre dans les communautés vient aussi de cette bataille pour la survie et les enfants sont tous associés à cette bataille. Depuis une dizaine d'années, les viols de femmes et de jeunes filles ne sont plus les seules violences sexuelles enregistrées. Des viols de bébés et de jeunes enfants (uniquement des fillettes) sont apparus dans certains villages autour de Bukavu toujours en toute impunité. Ces villages sont entourés de milices, mais aussi de forces armées et de la Monusco. Le modus operandi de ces viols est connu : les petites sont enlevées, endormies dans la case familiale, et rejetées, ensanglantées à proximité ou sur le seuil de la maison. On ignore le mobile de ces crimes. S'agit-il de terroriser le village ou de collecter du sang pour des militaires ou miliciens afin de satisfaire à d'obscures croyances d'invulnérabilité ou de puissance au combat grâce à ce sang virginal ? Certaines de ces victimes meurent, d'autres parviennent à survivre grâce à la chirurgie réparatrice du Docteur Mukwege à Panzi. Panzi est en effet le seul hôpital de la région à opérer par laparoscopie. Cette technique chirurgicale est moins invasive et permet d'éviter au bébé le choc d'une incision trop grande alors que le viol a été dévastateur.

Dès que la petite victime s'est remise de son intervention chirurgicale, elle rentre avec sa mère dans son village. C'est sur cette dernière que repose le soin de nourrir la famille et elle ne peut s'en éloigner longtemps. Les petites filles violées n'ont donc pas la possibilité matérielle de bénéficier des soins prodigués par Panzi en cas de viol. L'hôpital fonctionne en effet sur base d'un modèle holistique développé par le Docteur Mukwege. Le modèle tient compte de la globalité des problèmes rencontrés en cas de viol : on y trouve un pilier économique avec le développement des moyens de survie des survivantes de violences sexuelles grâce à de la formation professionnelle et des microcrédits, un pilier juridique (mise sur pied d'une clinique juridique) pour favoriser le retour à un état de droit, grâce à la lutte contre l'impunité ; un pilier reconstruction du corps, avec les soins gratuits fournis par l'hôpital de Panzi aux victimes ; un pilier reconstruction du psychique avec les soins psychologiques fournis aux victimes par les psychologues de Panzi.
Mais les fillettes qui rentrent immédiatement au village vont devoir, comme l'attestent la littérature scientifique et l'expérience dans d'autres zones de conflit, faire face à des séquelles durables. En 10 ans, Panzi a brièvement accueilli plus de trois-mille cinq mineures violées, soit environ une par jour, et parmi elles, environ 2500 enfants de moins de dix ans. C'est sans compter les décès et les fillettes soignées dans d'autres hôpitaux locaux, qui n'ont pas eu de chirurgie réparatrice et ont des séquelles physiques intolérables (fistules, infections urinaires, incontinence, etc.). Mal connue, dispersée dans différents villages, cette population d'enfants violées - privées de leur enfance, parfois privées de toute instruction, de soins médicaux et d'accompagnement psychologique - a un besoin urgent d'aide.

\section{La prise en charge thérapeutique et les sites d'action}

EPA a entamé dès 2016, la prise en charge de 63 fillettes de moins de dix ans, violées dans 3 villages autour de Bukavu. Cette prise en charge se fait en partenariat avec la Fondation Panzi, bâtie dans l'enceinte de l'hôpital du même nom. Elle a notamment consisté en l'organisation de jeux thérapeutiques collectifs, mêlant à part égale des enfants violées et d'autres qui ne le sont pas pour éviter toute stigmatisation des victimes. L'aide de " mamans " pour préparer les collations lors des jeux a été sollicitée afin d'intégrer la communauté locale. Les écoles ont été associées à cette dynamique, et les frais de scolarité des enfants les plus pauvres ont été pris en charge par EPA. La thérapie par le jeu est aujourd'hui largement utilisée pour reconstruire psychologiquement les enfants victimes de stress post traumatique [2-6]. Sans pathologiser leur état, sans éloigner les enfants de leur communauté, elle permet en effet l'expression des émotions, de la souffrance latente, le retour à la socialisation et à la confiance dans un environnement bienveillant. C'est une porte ouverte aussi sur l'imaginaire, sur un avenir meilleur dans lesquels les enfants peuvent se projeter, eux dont la vie semblait figée sur l'événement traumatique du viol. Durant l'année 2017, des jeux thérapeutiques ont été organisés deux fois par mois sur les trois sites par la coordinatrice et les trois assistantes sociales affectées aux différents sites. À côté de cette prise en charge collective, un suivi individualisé et à domicile des familles des victimes a été assuré et une série de données a été recueillie pour suivre l'évolution des enfants.

\section{Méthodologie et hypothèses de travail}

Une série de données considérées comme des variables « indépendantes " a été recueillie à propos de chaque fillette : âge actuel, 
âge au moment du viol, opération chirurgicale après viol ou pas, niveau de pauvreté de la famille, scolarisation ou pas, présence ou non du père au foyer. En tout, 13 variables de ce type ont été inventoriées.

L'évolution de l'enfant au cours de la thérapie par le jeu a été suivie grâce à un indice de fonctionnement global de l'enfant (le Pentagone d'équilibre) mis au point par le Prof. De Keyser de l'université de Liège, car d'autres indices ou échelles existants étaient, sur le plan culturel, inadéquats dans le contexte congolais. Le Pentagone comporte 5 axes : un axe sentiment de sécurité, un axe santé, un axe développement cognitif, un axe intégration au sein de la famille, un axe intégration dans la communauté. Chaque axe se décline à travers 5 questions ; l'assistante sociale y répond en fonction de son observation du comportement de l'enfant et des informations fournies par la famille lors de ses visites à domicile. Les scores l'aiguillent non pas vers un diagnostic de pathologie clinique particulière, mais vers des conseils psychopédagogiques à prodiguer aux mamans ou à la communauté. L'objectif est en effet d'éviter que ne soit collée aux enfants une étiquette de pathologie mentale, dont les interprétations par la famille et la communauté peuvent se révéler désastreuses. La croyance en l'existence d'enfants possédés, d'enfants dits sorciers, et le recours à des exorcismes ou des guérisseurs pour les désenvoûter restent une menace dans cette région de post conflit, marquée par la violence et la misère [7-9]. À partir des variables indépendantes et des mesures répétées du Pentagone d'équilibre pendant les six derniers mois de 2017, trois hypothèses de travail ont été posées :

- H.1. Les trois sites sont comparables sur le plan de l'échantillon d'enfants violés qui s'y rattachent ;

- H.2. La thérapie par le jeu améliore le fonctionnement global des enfants dans leur milieu ;

- H.3. Certaines variables « indépendantes » influencent la manière dont les enfants évoluent : par exemple la sévérité du viol, l'absence du père, l'absence de scolarisation, la pauvreté extrême de la famille, etc. sont attendus comme influençant négativement le fonctionnement global de l'enfant.

\section{Résultats}

4.1. Les trois "échantillons d'enfants" sont-ils a priori comparables, sur le plan de leurs caractéristiques individuelles?

Lorsqu'on explore l'âge des victimes et l'âge au moment du viol, la pauvreté des familles, la présence ou non du père, la stigmatisation, on ne trouve guère de différences significatives entre les échantillons des trois sites ([F2,59] = 0,14, $p=0,87$ ).

Les ressources des familles touchées par le viol sont très faibles : $33 \%$ ont moins de 10 dollars par mois, $43 \%$ vivent avec 11 à 20 dollars par mois, $10 \%$ ont de 21 à 50 dollars par mois et $14 \%$ ont plus de 50 dollars par mois. En fait, la conclusion de ce survol est terrible : $75 \%$ des enfants violées sont issues de grandes fratries qui survivent avec 20 dollars ou moins par mois et 1 tiers de l'échantillon des 57 familles a 10 dollars ou moins par mois (voir Tableau 1). La scolarisation des enfants est très importante, ce qui peut surprendre, mais les frais de scolarité de 2017 ont été pris en charge pour les enfants en grande difficulté financière par deux associations : EPA pour deux sites et une ONG espagnole, Coopera, pour le troisième. Le père est absent dans près de la moitié des familles. La stigmatisation de la famille au sein de la communauté touche $40 \%$ des familles des victimes.

La moyenne d'âge de l'échantillon des 57 victimes est de 7,7 ans (voir Fig. 1) et l'âge moyen au moment du viol est de 5,4 ans. Mais cette dernière moyenne cache une courbe bimodale avec deux moments clés : autour de l'âge de 4 ans et autour de l'âge de 8 ans
Tableau 1

État de quelques variables indépendantes dans les 3 sites.
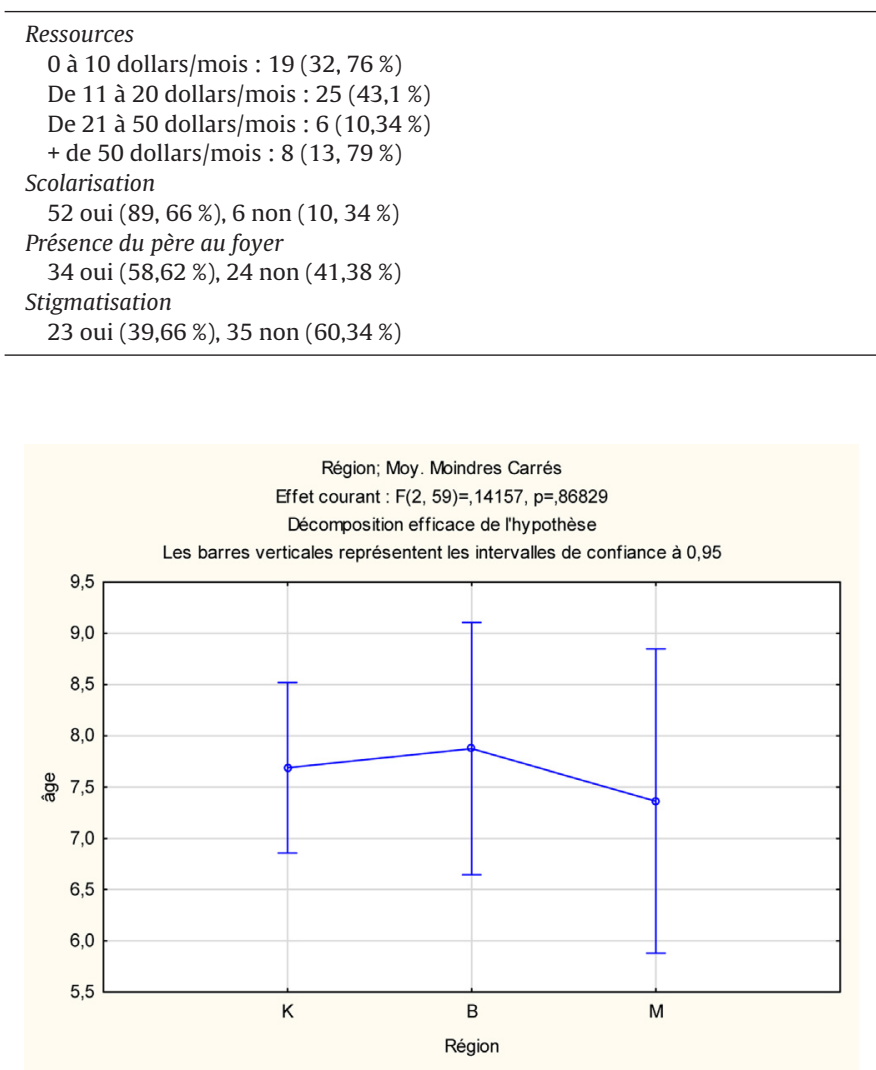

Fig. 1. Âge moyen des fillettes sur les trois sites, à K., B. et M. Pas de différence significative entre les trois sites ([F2,59] $=0,14, p=0,87)$.

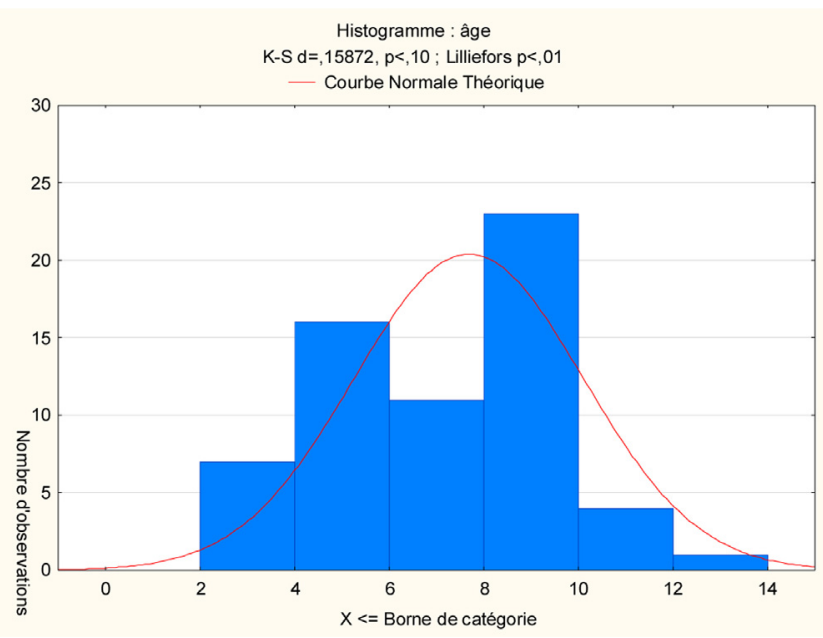

Fig. 2. Âge moyen du viol, courbe bimodale avec effet significatif de l'âge.

(voir Fig. 2). Ce sont les âges où les enfants sont les plus exposés au viol.

La conclusion de l'analyse des variables indépendantes est que les trois échantillons sont homogènes pour l'ensemble ces variables. De plus il se révèle que la variable indépendante qui aurait pu être déterminante - à savoir la gravité du viol estimée à travers l'intervention chirurgicale qui l'a suivi - n'est pas pertinente pour prédire ou expliquer l'état psychologique de l'enfant. 


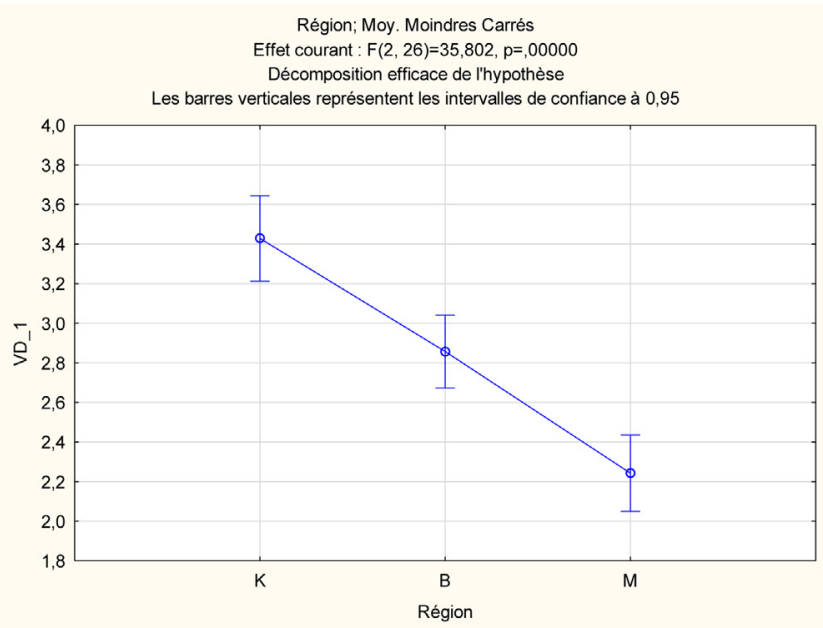

Fig. 3. Moyenne des résultats de l'axe sécurité dans chacun des trois sites, K, B et M. À noter que les cotes les plus élevées révèlent les perturbations de l'enfant les plus importantes. Les barres verticales représentent l'intervalle de confiance autour de la moyenne. La différence entre les 3 sites est significative.

\subsection{Incidence des jeux thérapeutiques sur l'équilibre de l'enfant et effet de contexte}

Cette exploration statistique permet de voir si les enfants semblent bénéficier de la prise en charge thérapeutique par le jeu. Le Pentagone d'équilibre a été utilisé pour définir l'état de l'enfant et le suivre, par des mesures répétées chaque mois, de mai à décembre 2017.

Dans les trois sites, l'axe qui présente le plus de perturbation est l'axe sécurité, puis l'axe santé. Les axes développement intellectuel, intégration familiale et intégration sociale sont dans des limites acceptables. À noter que la cotation de chaque axe va de 1 à 5 . Un score de 5 est le déséquilibre maximal et 1 l'optimal. Toute cote à partir de 3 est considérée comme préoccupante.

\subsubsection{L'axe sécurité}

La Fig. 3 témoigne d'une différence significative entre les trois sites, non pas au départ de la prise en charge, ce qui est important, mais six mois plus tard, fin décembre $2017(F[2,26]=35,8$, $p<0,000)$. La moyenne sur l'axe sécurité des enfants de $\mathrm{K}$. est significativement plus élevée que celle de B. qui est elle-même significativement supérieure à $\mathrm{M}$.
Comme en témoigne la Fig. 4, les tendances marquées par M. et K. fin 2017 sont loin d'être encourageantes. K. se dégrade lentement et finit à près de 4 de moyenne alors que $B$. s'améliore au cours du temps de façon spectaculaire et termine en fin d'année à moins de $2(\mathrm{~F}[12,156]=12,2, p<0,00)$. L'effet de contexte que suggèrent ces résultats sera commenté dans la discussion. Il faut noter que les jeux varient chaque mois, mais sont les mêmes dans les trois sites, avec la même coordinatrice psychologique. L'effet enregistré n'est donc pas lié à la méthode thérapeutique utilisée, ni aux intervenants sur place.

\subsubsection{L'axe santé}

L'axe santé suit la même évolution que l'axe sécurité. Il y a une différence de moyennes entre les trois sites $(F[2,27]=7,34$, $p<0,005$ ), et une évolution au cours des mois qui atteste une amélioration notable de $B$., et une dégradation de $\mathrm{M}$ et de $\mathrm{K}$ $(\mathrm{F}[12,138]=4,48, p<0,00)$. À noter que l'axe santé n'est pas un axe rempli par un médecin et n'a pas de visée diagnostique : il tient compte des plaintes de la mère et de l'enfant : céphalées, maux de ventre, fièvres fréquentes, etc. Les symptômes peuvent être d'ordre psychosomatique.

Sur le plan des moyennes, les autres axes ne présentent pas de scores moins inquiétants et leur évolution n'est pas significative. Mais les moyennes ne sont que des indicateurs très grossiers.

\subsection{Certaines variables indépendantes affectent-elles l'évolution de l'enfant? Y-a-il un effet qui pourrait s'apparenter à un effet médiateur, accentuant ou minimisant l'effet du viol ?}

Peut-on imaginer que la sévérité du viol, l'absence de scolarisation, la présence ou non du père ou d'autres variables parmi les 13 relevées, jouent sur l'équilibre de l'enfant, tel qu'appréhendé par le Pentagone d'équilibre ? On a souligné une première influence, celle du site (contexte géographique, sécuritaire et culturel) dont provient l'enfant. Voyons à présent l'influence possible de la présence du père dans la famille.

La présence du père dans la famille (rappelons qu'un tiers des enfants vivent dans des familles où le père n'est pas présent) a un effet positif sur le sentiment de sécurité de l'enfant quel que soit le site, mais cet effet n'est pas significatif $(F[1,23]=2,53, p=0,14)$. Cette différence est plus grande à $\mathrm{M}$. et à $\mathrm{B}$., contrairement à $\mathrm{K}$. où l'insécurité y est telle que la présence du père ne semble pas pouvoir l'atténuer (voir Fig. 5).

Pour les autres variables, à part la présence du père qui influe légèrement sur l'axe sécurité, il ne semble pas y avoir de relation. Cette absence de relation est un résultat en soi. Ainsi, les résultats

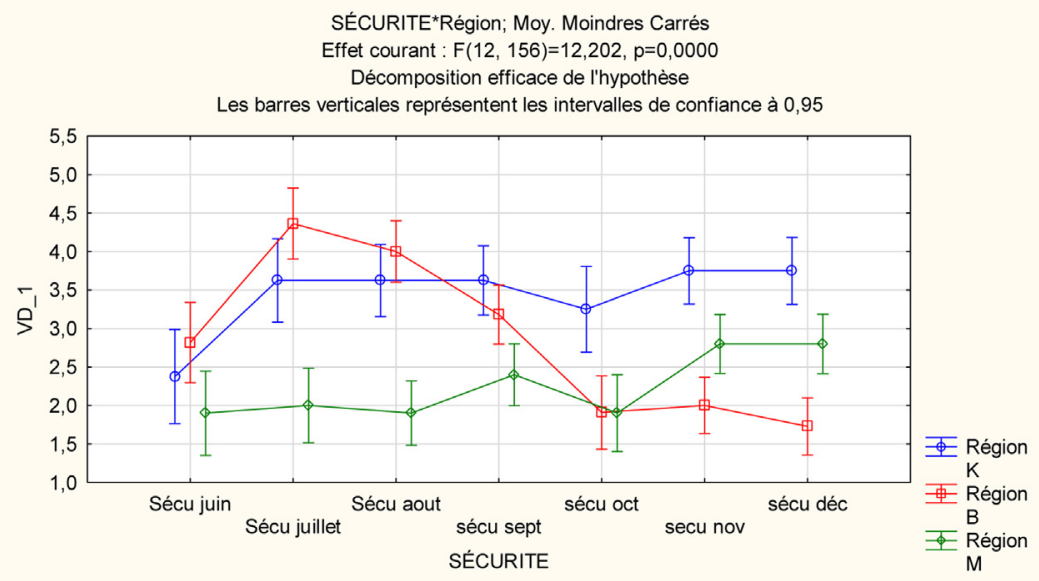

Fig. 4. Évolution de l'axe sécurité dans les trois sites sur les 6 mois de recueil des données. La cote la plus élevée signe la perturbation la plus importante. 


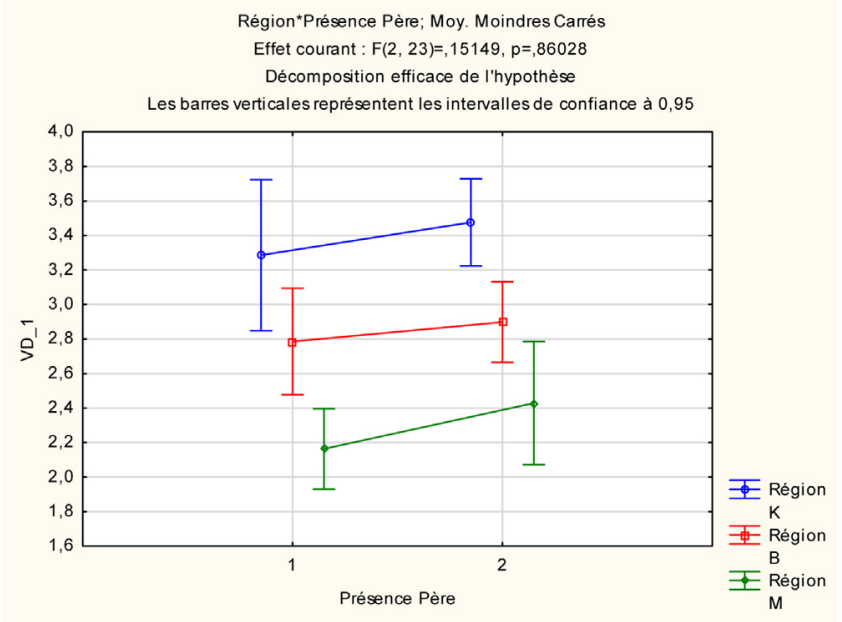

Fig. 5. Effet de la présence du père sur le sentiment de sécurité de l'enfant dans les trois sites. Le sentiment de sécurité est en ordonnée : plus la note est élevée, plus l'insécurité est grande. La variable présence du père, en abscisse, est dichotomique : le 1 est la présence du père au foyer et le 2 l'absence.

comparant les enfants opérés vs les enfants non opérés au Pentagone d'équilibre ne sont pas différents $(F[6,162]=1,58, p=0,38$, notons en outre qu'à K., un grand nombre d'enfants a été opéré à Panzi). Pas plus que ceux des scolarisés vs les non-scolarisés $(\mathrm{F}[6,162]=1,12, p=0,52)$. La pauvreté des parents $(\mathrm{F}[6,162]=1,43$, $p=0,49)$ et la stigmatisation $(F[6,162]=0,79, p=0,57)$ pas davantage. Le viol, la pauvreté et sa possible stigmatisation sont devenus la norme. La lutte permanente pour la survie aussi. Ce point également fera partie de la discussion.

\subsection{L'existence d'un groupe à risque important : circonstances $d u$ viol ou dynamique familiale cachée?}

Les thérapies par le jeu ont été complétées par des visites régulières dans les familles des victimes. Les observations des assistantes psychosociales et celles de la coordinatrice EPA, ont fait apparaître que près de la moitié des fillettes présentent des symptômes cliniques inquiétants et persistants assimilables à des séquelles de stress post traumatique. Et ce dans les trois sites sans prédominance d'une région. Une rapide comparaison entre les résultats de Pentagone d'équilibre du groupe à risque et non à risque dans les trois sites, montre une différence significative entre ces deux groupes principalement dans l'axe sécurité et santé, mais aussi, partiellement dans l'axe développement intellectuel et intégration sociale. Ainsi, les fillettes identifiées comme cas complexes se montrent significativement plus fragiles que les autres sur le plan de la sécurité $(F[6,162]=8,82, p<0,01)$, de la santé $(F[6,162]=6,76$, $p<0,01)$, du développement intellectuel $(\mathrm{F}[6,162]=4,66, p<0,05)$ et de l'intégration sociale $(F[6,162]=3,78, p<0,05)$. Ce résultat ne s'explique pas par les variables indépendantes, même pas par la gravité du viol (opération ou pas) ; il doit être creusé, car il est capital dans la suite thérapeutique à donner au programme EPA. Ne pouvant nous prononcer sur la cause de cette dynamique endogène - s'agit-il de conséquences liées aux circonstances du viol ou d'une dynamique familiale cachée ? - nous appellerons ce résultat un " effet individuel » à affiner ultérieurement.

\subsection{Synthèse des résultats}

Les premières réponses que l'on peut faire aux hypothèses de travail énoncées sont donc assez claires :
- H1. Oui sur le plan des variables individuelles, les trois échantillons d'enfants sont très semblables. On peut donc les comparer. Et même s'il y a plus de cas " sévères " de viols à K., à savoir des viols ayant requis une intervention chirurgicale, ce dernier facteur ne semble pas avoir d'influence sur l'évolution de l'équilibre de l'enfant ;

- H2. La prise en charge psychologique de l'enfant par le jeu semble n'avoir été totalement positive que sur un site, celui de B. Le site de $\mathrm{M}$. montre une légère détérioration des scores au Pentagone d'équilibre. Dans le site de K. l'évolution des enfants a été négative. Une dynamique contextuelle particulière explique sans doute cette détérioration ;

- H3. À l'exclusion de la variable présence du père et de celle liée au site, aucune variable indépendante relevée n'est prédictive de l'évolution de l'enfant. Mais un groupe à risque, qui comprend près de la moitié des enfants (25 sur 57) a émergé à partir des observations cliniques de l'équipe psychosociale qui suit les enfants dans les familles. Proportionnellement au nombre d'enfants, il n'est pas plus important à K que dans les deux autres sites.

Deux conclusions émergent de cette exploration des données des six premiers mois de prise en charge d'une soixantaine de fillettes en bas âge violées au Sud Kivu : l'existence d'un facteur de contexte lié aux particularités du site et d'un facteur individuel ou intrafamilial. Ce dernier se révèle dans l'existence d'un groupe à risque important, qui résiste aux thérapies ludiques collectives.

L'effet de contexte est lié à l'histoire du site où vit l'enfant. L'effet individuel ou intrafamilial est lié à l'histoire de la fillette et de son viol et à la dynamique familiale qui l'a entourée. Pour l'instant nous avons peu d'information à ce sujet. Mais nos résultats montrent que la thérapie par le jeu n'a guère d'incidence sur l'effet individuel et à ce jour, guère d'incidence sur l'effet lié au site - même si les familles déclarent que les jeux sont importants pour leurs enfants et qu'elles trouvent que les fillettes vont mieux après. L'intérêt de cette thérapie collective cependant n'est pas nul, car c'est le point d'accès aux communautés, l'occasion de rencontres et d'une mixité entre enfants violées et non violées sur un terrain de jeu. C'est l'occasion de collations prises ensemble et d'un petit travail rémunéré assuré aux mamans du village qui les organisent. La fonction sociale des jeux thérapeutiques est donc importante dans le cadre de la lutte contre la stigmatisation.

Le tableau clinique préoccupant de près de la moitié des fillettes, appelé groupe à risque, est un résultat à prendre tout à fait au sérieux. Dans certains cas, des dynamiques familiales qui dysfonctionnent sont suspectées, car la maltraitance, le rejet, l'absence de soins et de nourriture affectent certaines fratries. Les causes de ces dynamiques intrafamiliales doivent être explorées plus attentivement - viol possible de la mère et d'autres membres de la famille, prostitution éventuelle liée à la misère, maladie de la mère, VIH, etc. Mais dans d'autres cas, le comportement de la fillette exprime de l'anxiété, un état de dissociation, un mutisme ou au contraire de l'agressivité et même chez de très jeunes victimes, une hypersexualité explicite. Ces symptômes suggèrent plutôt une problématique individuelle liée à la mémoire traumatique du viol. Un travail thérapeutique approfondi sur la relation mère-enfant et sur la construction d'une interprétation du viol et de ses circonstances sont indispensables. Ce travail nécessite d'abord un renforcement des capacités cliniques de l'équipe psychosociale locale. Cette condition préalable est en voie de réalisation. 


\section{Discussion}

\subsection{Le déni du viol d'enfants en bas âge}

En Afrique, comme en Europe, des violences sexuelles s'exercent sur des enfants en très bas âge. Dans l'enquête qu'elle a menée sur les abus sexuels aux mineurs en 2015 en France, le Dr Muriel Salmona montre que la moitié des victimes d'abus sexuels sont des mineures où, pour un tiers, ces viols se produisent avant l'âge de 5 ans [10]. Les cas de bébés restent cependant exceptionnels en Europe sauf quand ils sont liés à la perversion et son industrie pédopornographique. Mais en Afrique comme en Europe, l'idée d'un tabou sur les enfants fait partie du déni associé à ce phénomène. C'est ce tabou fragile, on le voit, et le déni de sa transgression, qui permettent au phénomène de perdurer, et aux criminels de jouir de l'impunité. Au Sud Kivu, la présence de milices et de forces armées basées dans la région depuis la fin du conflit [11], l'existence de croyances magiques liées au sang virginal de petites filles - sang qui concentrerait les chances de la vie, garantirait l'invincibilité ou protégerait du sida - sont autant d'incitants à des trafics lucratifs de sang dans une économie de survie. Des cas de prostitution enfantine contre nourriture ont également été recensés. Et l'absence de travail de mémoire sur les viols, crimes de guerre et crimes contre l'humanité dénoncés dans le rapport Mapping [1] ont totalement délité les liens sociaux et la norme morale des communautés. La violence sociale latente est palpable. La pauvreté endémique dont est frappée la population dans une région aux ressources minières prodigieuses en est un des éléments. Il est difficile de prendre en charge ces fillettes sans dénoncer en même temps les conditions extrêmes dans lesquelles elles grandissent et appeler à davantage de justice sociale et à une meilleure gouvernance politique. Celle-ci devrait protéger les plus vulnérables et faire du viol, de sa prévention, du traitement de ses séquelles, de la sanction des criminels et de l'indemnisation des victimes, un programme politique et une priorité de santé publique. Le plaidoyer entrepris par le Docteur Mukwege, prix Nobel de la paix 2018 et directeur de l'hôpital de Panzi, n'est pas extérieur au travail de suivi des fillettes sur le terrain : il en est au cœur.

\subsection{Quelles thérapies individuelles dans les villages?}

Pour de très nombreuses raisons, les prises en charge individuelles dans les villages sont compliquées. Les villages sont étendus, les routes peu sûres et difficiles d'accès. Les enfants du groupe à risque sont nombreux. Ils se déplacent une à deux fois par mois aux séances de jeux thérapeutiques, leur frais de transport sont payés - mais se rendre même une fois par mois à l'hôpital de Panzi est une expédition impensable vu son coût, l'insécurité des routes et le manque à gagner de la maman si elle s'absente de son foyer. C'est donc au sein même du village, et autour du socle que constituent les jeux thérapeutiques, qu'une prise en charge individualisée peut se construire. La durée de celle-ci et sa forme sont encore à moduler en fonction des contraintes contextuelles. La thérapie individuelle par le jeu, centrée sur l'abus sexuel comme la pratique Gavin [3] devrait donner de bons résultats. Elle prolongerait l'approche collective utilisée jusqu'ici pour affronter plus directement la question du viol et de la mémoire traumatique, sans devoir repasser par la case hôpital de Panzi. Car l'ancrage de l'enfant dans sa communauté, et le soutien de cette dernière sont essentiels. Ils sont le contenant culturel qui sert de matrice à la signification donnée au viol, et la voie d'une possible réintégration de ce dernier dans la mémoire autobiographique de l'enfant [10]. Par sa fonction symbolique, la communauté est donc un partenaire essentiel de la thérapie, même lorsque celle-ci s'exerce à un niveau individuel.

\subsection{L'effet contexte dans le site de $\mathrm{K}$}

Dans le site de K., où la détérioration de l'équilibre des enfants se lit dans les résultats, ce soutien à la thérapie a été questionné et fait l'objet de tensions. Le village de $\mathrm{K}$ est un village martyr où des bébés ont été violés massivement. Plus encore qu'à B., l'insécurité y est maximale. Ce drame a provoqué un afflux d'ONG, intervenant souvent sans coordination auprès des victimes, qui pour les détraumatiser, qui pour les relaxer, qui pour les instrumentaliser. La réponse de la société civile locale a été à la hauteur du défi : les parents des victimes se sont rapidement constitués en associations pour centraliser les demandes d'intervention et les filtrer. Certaines associations, bien implantées à K., y sont restées actives. Mais en 2017, K. a été le théâtre d'un procès historique [12]. Un tribunal militaire y a jugé pour la première fois dans la région, un député et sa milice accusé d'avoir perpétré des viols d'enfants. Il n'existe pas de loi congolaise protégeant les plaignants : les familles des victimes ont donc été directement exposées à une campagne d'intimidation et de terreur, allant jusqu'à des tentatives de kidnapping et d'assassinat des fillettes avant le procès. Certaines familles ont retiré leur plainte, d'autres ont fui définitivement le pays par crainte de représailles. Les familles qui avaient tenu bon et sont allées jusqu'au bout du procès n'ont cependant pas encore reçu, contrairement à leur attente, d'indemnisation du tribunal. Elles se sont donc retournées vers les ONG sur place en espérant y trouver des compensations financières, souvent impossibles à assurer. La communauté de $\mathrm{K}$. a revécu la stratégie des viols de guerre dont elle a été victime, si présente encore dans toutes les mémoires. Dans chaque enfant violé, c'est elle qui se sentait la cible et la première victime. Malgré les collectifs de parents, les associations de la société civile, les marches de la colère, elle n'est pas parvenue, durant la période explorée dans cette étude, à assurer son rôle de référent et de soutien aux petites survivantes. Ce va et vient de l'individuel au collectif, matrice de toute signification symbolique dans une société dominée par le social, n’a longtemps été à K. qu'un long chemin du sang. Aujourd'hui pourtant, EPA a réussi, après de patientes concertations et une réflexion sur sa propre pratique, à nouer des liens solides avec cette communauté meurtrie et à en faire le pilier d'une reconstruction psychologique des enfants. Un memorandum fixant les relations entre la communauté de K. et EPA a été signé : il n'a pas d'incidence financière sur les associations de familles et des collectivités locales, mais fixe les engagements des uns et des autres dans la thérapie des enfants à risque et renforce l'alliance thérapeutique.

\section{Conclusion}

Bien conscientes que la prise en charge psychologique des fillettes ne peut se faire que dans la durée et que six mois d'observation ne suffisent pas, ces premiers résultats ont conduit les auteures à revoir leur dispositif expérimental et leur suivi de la façon suivante.

En 2018, les séances de jeu thérapeutiques ont été réduites de deux séances par mois à une séance, pour dégager du temps et des ressources humaines pour la formation du staff à des approches thérapeutiques individuelles.

Le Pentagone d'équilibre n'était qu'un outil d'analyse fonctionnelle. Vu l'importance du groupe à risques, il a été décidé de mener une exploration diagnostique plus approfondie en confrontant trois groupes : le groupe à risque, le groupe des fillettes qui participent aux jeux thérapeutiques sans être victimes de violences sexuelles, et le groupe des victimes qui ne présentent pas de signes cliniques de perturbation. Une formation en ce sens de l'équipe psychosociale est en cours. L'accord des parents et celui de leur communauté sera requis. Parallèlement à ces données quantitatives, une anamnèse 
approfondie de chaque cas de viol et de ses circonstances sera faite au sein des familles.

Dans les investigations plus approfondies, un soin particulier est accordé à la relation mère-enfant-environnement social et à la dynamique communautaire. Si la communauté en effet joue un rôle de référent symbolique qui imprègne toute signification du viol pour l'enfant, ce référent est susceptible d'être travaillé : il doit être respecté, mais pas sacralisé. En effet, certaines pratiques utilisées pour laver l'honneur de la famille et celui de la victime (par exemple : médiation financière avec les familles de l'ordre de quelques dollars, obligation du violeur d'épouser sa victime, scène du viol répétée, mais cette fois devant toute la communauté pour laver la tâche) sont incompatibles avec l'objectif de faire de ces enfants martyres des femmes épanouies, ayant en main les clés de leur avenir.

Enfin, nous soulignons ici l'importance de l'évaluation continue des pratiques psychologiques utilisées sur le terrain, en particulier dans les situations extrêmes rencontrées au Sud Kivu. Des facteurs culturels et des stéréotypes peuvent biaiser l'approche de psychologues européens - mais les croyances des communautés elles-mêmes ne peuvent être sanctuarisées. L'exemple des exorcismes réalisés sur des enfants considérés comme sorciers, car malades, traumatisés ou simplement difficiles en est l'évidence. La relation « juste " à établir avec les communautés est affaire de petits pas, de réflexion et de confiance mutuelle : c'est un travail en profondeur et dans la durée, pour renforcer l'alliance thérapeutique et ses effets bénéfiques.

\section{Remerciements}

Ce travail n'aurait pu être réalisé sans le soutien financier du Ministère belge des Affaires Étrangères belge (programme Peace
Building), de Wallonie-Bruxelles International, de la Fédération de Wallonie-Bruxelles, de la Loterie nationale et de la Fondation roi Baudouin. Nous les en remercions.

\section{Déclaration de liens d'intérêts}

Les auteurs déclarent ne pas avoir de liens d'intérêts.

\section{Références}

[1] Rapport sur les violations sévères des droits de l'homme commises entre 1993 et 2003 en RDC, et commandé par N. Pillay, Haute commissaire des Nations Unies aux droits de l'Homme et rendu public en août; 2010.

[2] Jernberg A, Booth P. Theraplay. Une nouvelle thérapie par le jeu basée sur l'attachement. De Boeck; 2010 [Trad. 2014. Trad. F. Hallet].

[3] Gavin VG. La thérapie individuelle par le jeu et la créativité. In: Cyrilnik B, Seron C, editors. La résilience ou comment renaître de sa souffrance. Ed. Fabert; 2003.

[4] Sunderland M. Utiliser des histoires contées comme outil thérapeutique avec les enfants. De Boeck; 2016.

[5] Cariou-Rognant AM, Chaperon AF, Duchesne N. L'affirmation de soi par le jeu de rôle. Dunod; 2014.

[6] Klinkhamer-Steketee HT. Psychothérapie par le jeu. Mardaga; 1997.

[7] Cimpric A. Les enfants accusés de sorcellerie. Étude anthropologique des pratiques contemporaines relatives aux enfants en Afrique. Rapport Unicef. Dakar: Bureau Afrique de l'Ouest et du Centre (BRAOC); 2010.

[8] De Keyser V. Les enfants accusés de sorcellerie en Afrique Subsaharienne. Acte du colloque du 15 juin 2011. Parlement européen, Bruxelles; 2011.

[9] De Boeck P. Le « deuxième monde » et les « enfants sorciers». Politique Africaine 2000;80:32-57.

[10] Salmona M. Le livre noir des violences sexuelles. Dunod; 2018.

[11] Fouchard I. Violences sexuelles commises par le personnel des Nations Unies tolérance zéro, impunité UN. In: Cazala J, Lecuyer Y, Taxil B, editors. Sexualité et droit international des Droits de l'homme. 13, rue Soufflot, 75005 Paris: Ed. A. Pedone; 2018.

[12] Naimer K, Volpellier M, Mukwege D. The case of Kavumu: a model of medicolegal collaboration. Lancet 2019;393:2651-4. 